\title{
INFLUENCIA DEL INJERTO Y LA RELACIÓN POTASIO/NITRÓGENO SOBRE EL RENDIMIENTO Y CALIDAD DEL TOMATE VALENCIANO
}

\author{
J. Lambies, A. San Bautista, S. López* \\ Departamento de Producción Vegetal, ETSIAMN, \\ Universitat Politècnica de València \\ *slopez@upv.es
}

\section{Resumen}

En el presente trabajo se estudió la influencia del injerto (plantas injertadas en el portainjerto 'Maxifort' (L. esculentum x L. Hirsutum), plantas auto-injertadas y plantas sin injertar) y de dos soluciones nutritivas con diferentes ratios $\mathrm{N} / \mathrm{K}(1.3$ y 1.8$)$ en parámetros de producción y calidad del fruto en la variedad tradicional de tomate valenciano. El efecto del injerto no se hizo notar en la producción total pero sí en la distribución de los calibres, produciendo las plantas injertadas un porcentaje mayor de frutos de primera y un menor porcentaje de frutos de segunda y tercera respecto de las plantas sin injertar. No se encontraron diferencias en el número total de frutos por planta, pero si en el peso medio total del fruto, siendo mayor en las plantas injertadas. No se observaron diferencias significativas en la presencia de fisiopatías. Respecto al efecto de la solución nutritiva, el equilibrio $\mathrm{N} / \mathrm{K}=1.3$, dio lugar a una mayor producción de frutos de primera no observándose diferencias en el resto de los calibres ni en la presencia de fisiopatías. No se encontraron diferencias significativas en los parámetros de calidad ni en el factor injerto ni en el factor solución. El uso del injerto y el manejo de solución nutritiva pueden ser dos buenas herramientas a la hora de aumentar el porcentaje de frutos de primera en tomate valenciano sin que se vean afectadas sus características de calidad.

Palabras clave: portainjertos, tomate valenciano, productividad, ratio N/K.

\begin{abstract}
In the present work we studied the influence of rootstock (plants grafted onto rootstock 'Maxifort' (L. esculentum x L. hirsutum), self-grafted
\end{abstract}


plants and non-grafted plants) and two nutritive solutions with different $\mathrm{N} / \mathrm{K}$ ratios (1.3 and 1.8) in productivity and fruit quality parameters of the traditional Valencian tomato variety. The gratfing did not have an effect on total yield, but was significant for distribution of fruit size categories, so that that grafted plants had a higher porcentaje of first category and a lower percentage of second and third category compared to the non-grafted plants. No differences were found for the total number of fruits per plant, but these were significant for the average weight of the fruit, which was higher in the grafted plants. No significant differences were observed for physiological diorders. Regarding the nutritive solution effect, the $\mathrm{N} / \mathrm{K}=1.3$ equilibrium gave a higher yield of first category fruits, with no differences being observed for the rest of categories or in the presence of physiological disorders. No significant differences were found for quality parameters neither for the grafting factor nor for the solution factor. The use of grafting and management of nutritive solution can be two tools to increase the percentage of first category fruits in Valencian tomato, without having the quality characteristics being affected.

Keywords: rootstocks, valencian tomato, productivity, N/K ratio.

\section{1.- INTRODUCCIÓN}

El tomate valenciano incluye a uno o varios ecotipos tradicionalmente cultivados en la huerta de Valencia, cuyos frutos se caracterizan por poseer unas características cualitativas externas e internas excepcionales y de ahí su creciente valoración por los consumidores, no sólo valencianos. Estas características cualitativas se ensalzan además cuando son cultivados en determinadas condiciones de suelos y climas, por lo que en ocasiones se sub-clasifican estos ecotipos según localizaciones concretas. Sin embargo, su cultivo es exigente en nutrición y manejo. Por una parte, condicionado por la diversidad en el material vegetal, que ocasiona diferencias notables en rendimientos y en calidades comerciales, cuando se comparan con cultivares híbridos, mucho más uniformes (Cebolla, 2005). Por otra parte, por la interacción del material vegetal con las características del medio de cultivo, características edáficas y climáticas, particularmente cuando se cultiva en invernadero. Y, por último, por la escasa tolerancia de estos genotipos a los estreses tanto bióticos como abióticos. Todos ellos condicionan en gran medida sus resultados productivos, puestos de manifiesto en diversos estudios agronómicos (López y Gil, 2010; Giner et al., 2011). 
El injerto de tomate sobre patrones resistentes o tolerantes a diversos estreses es una estrategia productiva ampliamente instaurada en España y otros países de la cuenca mediterránea por mejorar significativamente los resultados económicos de la variedad cultivada, al menos en un contexto de producción ambientalmente respetuosa y frecuente repetición de cultivo (Hoyos 2007), aunque las diferencias entre los portainjertos pueden ser también notables.

El uso de híbridos interespecíficos como portainjertos ha mostrado una mejora en el desarrollo de la planta y en las producciones de tomate (Lee and Oda, 2003; Leonardi and Giuffrida, 2006; Colla et al., 2008, 2010; Di Gioia et al., 2010; Djidonou et al.,2013).

Para tomate se utilizan principalmente híbridos interespecíficos de Solanum lycopersicon $\mathrm{x} S$. habrochaites y $S$. lycopersicon $\mathrm{x} S$. pimpinellifolium. También se emplea $S$. lycopersicon, aunque no son tan vigorosos como los híbridos interespecíficos (Miguel, 2011).

El injerto sobre patrones vigorosos ha conseguido incrementar entre un 20 y un $62 \%$ la producción de tomate comercializable, dependiendo de la combinación portainjerto-variedad y las condiciones de cultivo, frente a plantas no injertadas (Di Gioia, et al., 2010; Lee and Odal, 2003; Leonardi y Giuffrida, 2006). La utilización de injertos en variedades tradicionales también está aumentando (Rivard y Louws, 2008).

Los parámetros de calidad más estudiados, sólidos solubles y acidez titulable, se han visto afectados por el uso de injerto con resultados dispares. En el trabajo de Flores et al. (2010) las plantas de tomate injertadas produjeron frutos con un nivel mayor tanto de ácido ascórbico como de sólidos solubles, mientras que en el trabajo de Barret et al (2012) no se encontraron diferencias en estos parámetros entre plantas injertadas y no injertadas.

El efecto del injerto en tomate ha resultado en muchos casos en un aumento tanto del número de frutos como en su peso medio, lo que aumentó la producción total de las plantas injertadas frente a las no injertadas. (Turhan et al., 2011; Gebologlu et al., 2011; Khah et al. ,2006; Ibrahim et al., 2014). En concreto, en un trabajo de la Fundación Cajamar (Giner et al., 2011), se estudió el comportamiento de trece portainjertos de diferentes casas comerciales en la variedad tradicional de tomate valenciano. Varios de estos portainjertos superaron en producción a las plantas testigo. También 
redujeron el porcentaje de destrío al disminuir la incidencia de "blossom-end rot" (BER), cicatriz estilar ("cat face") y el rajado de frutos ("cracking").

Por otra parte, es bien conocida la repercusión de las variaciones en la fertilización sobre los rendimientos del tomate en función de los cultivares empleados y particularmente de las relaciones entre las aportaciones de potasio y nitrógeno no sólo sobre el cuajado de frutos sino también sobre el tamaño final de los frutos.

Numerosos experimentos han demostrado que existe una marcada interacción entre el Nitrógeno y el Potasio (Johnston y Milford, 2012). Para obtener cosechas altas y de calidad se deben aplicar óptimas proporciones $\mathrm{N}: \mathrm{K}$. Altos ratios $\mathrm{N}: \mathrm{K}$ no necesariamente conllevan un incremento en la cosecha e incluso pueden reducirla (Zhang et al., 2010). Según Chaux (1972), citado por Maroto (2002), los ratios N/K deben variar según el estado fenológico de la planta. En las primeras aportaciones, la proporción $\mathrm{N} / \mathrm{K}$ será de 1:2; cuando se esté produciendo la floración del tercer-cuarto racimo, esta proporción se situará en $1: 1$, y en plena maduración esta proporción se elevará de nuevo a 1:2.

Algunos trabajos han intentado encontrar este ratio óptimo para el cultivo de tomate en producción y calidad. En algunos casos la variación de la relación N/K en la solución nutritiva influyó en el rendimiento, la calidad externa y la vida en almacén de los frutos de tomate, sin afectar la calidad bromatológica (Hernández et al, 2009), siendo la mejor combinación para el rendimiento y calidad de los frutos la relación N/K 1:0.75. En otros estudios los mejores resultados se encontraron con un ratio N/K 1:1,2 (Iqbal, 2011).

Con el fin de proporcionar una adecuada fertilización nitrogenada y potásica es muy común el uso de nitrato potásico, pero en algunos estudios se demostró que se puede utilizar el cloruro potásico como alternativa a este fertilizante mejorando algunos parámetros de calidad como una reducción de podredumbres y manchados en el fruto sin que hubiera diferencias ni en materia seca, ni en sólidos solubles, ni en acidez valorable comparado con el tratamiento con nitrato potásico (Chapagain et al.., 2003).

Los resultados que se presentan en esta aportación son fruto de un experimento, realizado en condiciones controladas, en el que se estudió la influencia del injerto y la relación potasio/nitrógeno en la solución nutritiva, sobre los rendimientos y la calidad del tomate valenciano, tratando de evaluar las posibles interacciones entre ellos. 


\section{2.- MATERIAL Y MÉTODOS}

Este estudio se realizó en un invernadero de vidrio de $6 \times 24 \mathrm{~m}$, tipo Venlo, de la UPV ( $39^{\circ} 38^{\prime} 2^{\prime}$ ' $\mathrm{N}, 0^{\circ} 22^{\prime} 29^{\prime}$ ' O), dotado de ventilación cenital y sistema de control climático, con un sistema "cooling system".

El estudio se realizó con una selección de tomate 'Valenciano' injertada sobre el porta-injertos 'Maxifort' (De Ruiter Seeds) (hibrido interespecífico $S$. lycopersicon $x S$. habrochaites). La siembra y los injertos se realizaron por Viveros Cucala S.A (Benigànim, Valencia).

Las plantas fueron trasplantadas el día 16 de marzo de 2015 en contenedores de $8 \mathrm{~L}$ empleándose como sustrato una mezcla de turba rubia (35\%), turba negra (35\%), fibra de coco (15\%) y perlita $(15 \%)$, y se condujeron a un solo brazo.

Las plantas se fertirrigaron para satisfacer una fracción de agotamiento del $10 \%$ del agua fácilmente disponible en el sustrato y obtener una fracción de lavado del 15-25\% del agua aportada, variando la frecuencia de riegos de acuerdo con la radiación incidente. Las soluciones nutritivas suministradas, uno de los factores en estudio fueron las que se muestran en la siguiente tabla (valores en $\mathrm{mmol} \mathrm{L}^{-1}$ ). Diariamente se controlaba el volumen de la solución drenada y la CE de la misma. Hasta el día 20 de abril todas las plantas se regaron con la $\mathrm{S} 1$, y posteriormente se emplearon las dos soluciones nutritivas.

\begin{tabular}{|l|c|c|c|c|c|c|c|c|c|c|c|c|}
\hline & $\mathbf{N} / \mathbf{K}$ & $\mathbf{C E ~ d S m}^{-1}$ & NO3- & H2PO4- & SO42- & HCO3- & Cl- & NH4+ & K+ & Ca2+ & Mg2+ & Na+ \\
\hline S1 & $\mathbf{1 , 8}$ & 2,21 & 12,5 & 1,2 & 2,8 & 0,5 & 2,8 & 0,6 & 7 & 3,25 & 3,35 & 1,3 \\
\hline S2 & $\mathbf{1 . 3}$ & 2,26 & 8,5 & 1,2 & 2,8 & 0,5 & 6,8 & 0,6 & 7 & 3,25 & 3,60 & 1,3 \\
\hline
\end{tabular}

De cada combinación estudiada, tipo de planta -plantas sin injertar (NI), plantas injertadas en su propia variedad (SF) y plantas injertadas en el porta-injertos 'Maxifort' (IN)-, y solución nutritiva empleada -relaciones $\mathrm{N} / \mathrm{K}$ de 1,8 y 1,3-, se realizaron tres repeticiones de 10 plantas en un diseño completamente aleatorizado.

Las macetas fueron dispuestas en líneas pareadas con una distancia de $0.30 \mathrm{~m}$ entre plantas y $0,65 \mathrm{~m}$ entre líneas, con una distancia de $1,10 \mathrm{~m}$ entre las líneas pareadas, siendo la densidad de plantación fue de 3.3 plantas $\mathrm{m}^{-2}$.

En la plantación se regaron las macetas con agua sin solución nutritiva hasta alcanzar la capacidad de campo, después la cantidad de riegos 
aplicados fue cambiando en función de la radiación solar incidente para mantener un porcentaje de drenaje entre el 15 y el 20\%. Hasta el día 20 de abril todas las plantas se regaron con la $\mathrm{S} 1$ y posteriormente se emplearon las dos soluciones nutritivas.

El día 11 de mayo se despuntaron todas las plantas en la tercera hoja por encima del cuarto ramillete. La primera recolección se realizó el 25 de mayo y la ultima el 10 de julio.

Los frutos recolectados se dividieron en las siguientes categorías: primera (frutos $>200 \mathrm{~g}$ ); segunda $(150 \mathrm{~g}<$ frutos $<200 \mathrm{~g}$ ); tercera (frutos $<$ $150 \mathrm{~g}$ ), frutos con "blossom-end rot" (BER); frutos 'cat-faced' (frutos con presencia de cicatriz pistilar e invaginaciones en la zona apical); y frutos con 'cracking' tanto concéntrico como longitudinal.

En cada recolección y para cada unidad de repetición se tomaron frutos comerciales para las mediciones de parámetros de calidad. Se realizaron un total de 8 mediciones en las fechas 2, 17, 22,25 y 30 de junio y 2, 7 y 9 de julio. Loa parámetros medidos fueron:

- Densidad: un fruto por repetición se pesó y posteriormente se sumergió en un recipiente lleno hasta el borde con agua, calculando el volumen del fruto por el volumen desalojado de agua al sumergirlo. Conociendo el peso y el volumen, se calculó la densidad.

- Porcentaje de materia seca en el fruto: Un fruto por repetición fue pesado, troceado y secado en una estufa a $65^{\circ} \mathrm{C}$ hasta peso constante.

- Acidez titulable y $\mathrm{pH}$ inicial: Para la determinación de la acidez y el pH inicial se utilizó el método oficial 942.15 de la AOAC para zumos de fruta (AOAC, 2005)

- Sólidos solubles: Para la cuantificación de los sólidos solubles se determinaron los ${ }^{\circ}$ Brix con un refractómetro digital (Atago Palette, PR-32).

- Jugosidad: Se tomó el peso de un tomate por repetición. Posteriormente se exprimió el contenido con la ayuda de un exprimidor eléctrico. La pulpa obtenida se depositó en un colador durante 30 minutos, sin ejercer presión sobre ella, recogiendo en un vaso de precipitados el líquido filtrado, calculando la jugosidad como el cociente entre el peso del filtrado y el peso total del tomate 
Los datos obtenidos se sometieron a un análisis factorial de la varianza, empleando para ello el programa Statgraphics Centurion XVI, y mediante el test LSD $(\mathrm{p} \leq 0.05)$ se realizó la separación de las medias.

\section{3.- RESULTADOS}

\section{1.- Producción}

Analizando la producción acumulada por calibres hasta el cuarto ramillete se ha observado que las plantas injertadas (IN) produjeron más peso en frutos de primera $(\mathrm{p}<0.01)$ que las plantas auto-injertadas $(\mathrm{SF}), \mathrm{y}$ éstas produjeron más frutos de primera que las plantas sin injertar (NI) $(p<0.01)$. También se encontraron diferencias e.s. (estadísticamente significativas) en frutos de segunda y tercera, siendo las plantas IN las que menos peso produjeron en estos calibres respecto de las plantas SF y NI $(\mathrm{p}<0.05)$ (Tabla 1).

No se encontraron diferencias e.s. en la producción total acumulada entre los tres tipos de plantas.

Las plantas sin injertar produjeron más peso de frutos afectados por BER respecto de las SF y las IN $(\mathrm{p}<0.05)$.

La solución S2 dio lugar a una mayor producción por planta de tomates de primera que la solución $\mathrm{S} 1$. No se han encontrado diferencias para la producción de fruto de segunda y tercera. Para el resto de categorías no se obtuvieron diferencias e. s. entre soluciones nutritivas. Tampoco se encontraron diferencias e.s. en la producción total. 
Tabla 1.- Producción acumulada por planta por categorías comerciales, afectada por BER, 'cat-faced' y agrietados $\left(\mathrm{g} \mathrm{planta}^{-1}\right)$ y porcentaje acumulado de frutos (\%)

\begin{tabular}{|c|c|c|c|c|c|c|c|c|c|}
\hline & \multicolumn{3}{|c|}{ Producción acumulada } & \multicolumn{3}{|c|}{$\%$ acumulado de frutos } & \multirow[t]{2}{*}{$\overline{\mathrm{BER}}$} & \multirow[t]{2}{*}{ it-faced' } & \multirow[t]{2}{*}{ agrietados } \\
\hline & $1^{\mathrm{a}}$ & $2^{\mathrm{a}}$ & $3^{\mathrm{a}}$ & $1^{\mathrm{a}}$ & $2^{\mathrm{a}}$ & $3^{\mathrm{a}}$ & & & \\
\hline \multicolumn{10}{|l|}{$\overline{\text { Injerto (I) }}$} \\
\hline NI & $757 \mathrm{c}$ & $314.0 \mathrm{a}$ & $279.3 \mathrm{a}$ & $24.48 \mathrm{c}$ & 15.86 & $22.54 \mathrm{a}$ & $94.06 \mathrm{a}$ & 198.6 & 347.0 \\
\hline SF & $861 \mathrm{~b}$ & $318.4 \mathrm{a}$ & $264.0 \mathrm{a}$ & $29.67 \mathrm{~b}$ & 16.94 & $22.97 \mathrm{a}$ & $15.98 \mathrm{~b}$ & 583.6 & 196.2 \\
\hline IN & $1020 \mathrm{a}$ & $205.9 \mathrm{~b}$ & $149.3 \mathrm{~b}$ & $37.15 \mathrm{a}$ & 12.38 & $14.06 \mathrm{~b}$ & $30.35 \mathrm{~b}$ & 503.3 & 310.9 \\
\hline \multicolumn{10}{|l|}{ Solución (S) } \\
\hline $\mathrm{S} 1$ & $771 \mathrm{~b}$ & 285.8 & 253.6 & $27.39 \mathrm{~b}$ & 15.78 & 22.12 & 44.01 & 549.7 & 283.3 \\
\hline S2 & $987 \mathrm{a}$ & 273.0 & 208.1 & $33.47 \mathrm{a}$ & 14.34 & 17.59 & 49.58 & 507.3 & 286.1 \\
\hline \multicolumn{10}{|l|}{ Factor } \\
\hline Injerto & $* *$ & $*$ & $*$ & $* *$ & ns & $*$ & $*$ & ns & ns \\
\hline Solución & $* *$ & ns & ns & $* *$ & ns & ns & $\mathrm{ns}$ & ns & ns \\
\hline $\mathrm{I} \times \mathrm{S}$ & ns & ns & ns & ns & $\mathrm{ns}$ & ns & ns & ns & ns \\
\hline
\end{tabular}

NI: plantas no injertadas; SF: plantas autoinjertadas; IN: plantas injertadas. Letras diferentes en una columna indican diferencias e.s. con $\mathrm{p} \leq 0.05$. ns: no significativo; *: significativo $p \leq 0.05$; **: significativo $p \leq 0.01$

\section{2.- Peso medio acumulado por fruto}

No se han encontrado diferencias e.s. en el peso medio acumulado del fruto entre tratamientos, ni en el calibre, ni en fisiopatías.

Sin embargo, sí se encontraron diferencias e.s. en el peso medio total del fruto $(\mathrm{p}<0.01)$, siendo mayor el del fruto procedente de las plantas $\mathrm{IN}$ en comparación con los frutos de las plantas SF y NI. Entre estos dos últimos no se encontraron diferencias e.s.

No se observó una influencia de la solución en el peso medio acumulado del fruto por planta salvo en el caso de los frutos 'cat-faced', para los que la S2 dio un peso significativamente menor que en la S1 para esta fisiopatía $(p<0.05)$. No se observaron diferencias e.s. entre las dos soluciones en el peso medio total.

\section{3.- Número acumulado de frutos por planta}

Se obtuvieron diferencias e.s. en el número de frutos de primera, siendo superior en las plantas IN $(\mathrm{p}<0.01)$ con 3.61 frutos por planta, respecto de las SF con 3.16 frutos y las NI con 2.80 frutos por planta (Tabla 2). 
También se hallaron menos frutos de segunda y tercera en las plantas injertadas $(\mathrm{p}<0.05)$ respecto de los otros dos niveles del factor.

El número total de frutos por planta también se vio afectado por este factor, encontrando diferencias e.s. entre los tres tipos de plantas $(p<0.05)$, dando las plantas NI un mayor número de frutos respecto de las plantas SF e IN.

$\mathrm{Si}$ atendemos al efecto de la solución nutritiva observamos que las plantas que fueron regadas con la $\mathrm{S} 2$ produjeron más frutos de primera que la S1 ( $<<0.01)$, obteniendo 3.59 frutos de primera con la S2 frente a los 2.78 de la S1. No se vieron diferencias e.s. para este parámetro en el resto de categorías. Tampoco se encontraron diferencias e.s. en el número de frutos totales para este factor.

Tabla 2.- Número acumulado de frutos por planta y peso medio de los frutos (g) de cada categoría comercial

\begin{tabular}{|c|c|c|c|c|c|c|}
\hline & \multicolumn{3}{|c|}{ número de frutos } & \multicolumn{3}{|c|}{ peso medio } \\
\hline & $1^{\mathrm{a}}$ & $2^{a}$ & $3^{a}$ & $1^{\mathrm{a}}$ & $2^{a}$ & $3^{a}$ \\
\hline \multicolumn{7}{|l|}{ Injerto (I) } \\
\hline NI & $2.795 \mathrm{c}$ & $1.803 \mathrm{a}$ & $2.552 \mathrm{a}$ & 271.4 & 174.5 & 108.3 \\
\hline SF & $3.155 \mathrm{~b}$ & $1.815 \mathrm{a}$ & $2.473 \mathrm{a}$ & 273.2 & 174.8 & 107.3 \\
\hline IN & $3.608 \mathrm{a}$ & $1.178 \mathrm{~b}$ & $1.380 \mathrm{~b}$ & 282.4 & 173.8 & 106.9 \\
\hline \multicolumn{7}{|l|}{ Solución (S) } \\
\hline $\mathrm{S} 1$ & $2.778 \mathrm{~b}$ & 1.642 & 2.348 & 277.4 & 173.7 & 106.5 \\
\hline S2 & $3.594 \mathrm{a}$ & 1.556 & 1.922 & 274.0 & 175.0 & 108.4 \\
\hline \multicolumn{7}{|l|}{ Factor } \\
\hline Injerto & $* *$ & $*$ & $*$ & ns & ns & ns \\
\hline Solución & $* *$ & ns & ns & ns & ns & ns \\
\hline $\mathrm{I} \times \mathrm{S}$ & ns & ns & ns & ns & ns & ns \\
\hline
\end{tabular}

NI: plantas no injertadas; SF: plantas autoinjertadas; IN: plantas injertadas. Letras diferentes en una columna indican diferencias e.s. con $\mathrm{p} \leq 0.05$. ns: no significativo; *: significativo $\mathrm{p} \leq 0.05$; **: significativo $\mathrm{p} \leq 0.01$ 


\section{4.- Porcentaje acumulado de frutos por categorías comerciales}

El porcentaje acumulado de frutos en las categorías de primera, segunda y tercera siguió la misma tendencia que para el número de frutos por planta. En el caso de las fisiopatías, no se observaron diferencias e.s. entre los tres tipos de planta.

Respecto al efecto de la solución, se encontraron diferencias e.s. entre las dos soluciones, dando un porcentaje mayor de frutos de primera aquellas plantas que fueron regadas con la S2 frente aquellas a las que se aplicó la S1 $(\mathrm{p}<0.01)$.

\section{5.- Parámetros de calidad}

Solo se han observado diferencias e.s. en la densidad del fruto, siendo mayor en los frutos de las plantas IN que en los de las SF e NI $(\mathrm{p}<0.05)$ (Tabla 3).

Para el resto de parámetros no se hallaron diferencias significativas, pero se pudo apreciar que las plantas NI dieron valores ligeramente superiores en materia seca, acidez, grados ${ }^{\circ}$ Brix y jugosidad.

No se ha observó ningún efecto e.s. de la solución sobre los parámetros de calidad, si bien es cierto que las plantas regadas con la S2 dieron frutos con una acidez y unos ${ }^{\circ}$ Brix ligeramente superiores, así como un leve incremento en la jugosidad.

Las interacciones entre tipo de planta y solución nutritiva no fueron significativas en parámetro alguno. 
Tabla 3.- Parámetros cualitativos acumulados de la producción

\begin{tabular}{|c|c|c|c|c|c|c|c|}
\hline & $\begin{array}{l}\text { Densidad } \\
\mathrm{g} \mathrm{mL}^{-1}\end{array}$ & $\begin{array}{l}\text { Materia } \\
\text { seca } \\
(\%)\end{array}$ & $\mathrm{pH}$ & ${ }^{\circ}$ Brix & ${ }^{(1)}$ Acidez & $\begin{array}{l}{ }^{(2)} \text { Índice } \\
\text { madurez }\end{array}$ & ${ }^{(3)}$ Jugosidad \\
\hline \multicolumn{8}{|l|}{ Injerto (I) } \\
\hline NI & $1.016 \mathrm{a}$ & 5.201 & 3.892 & 4.365 & 0.515 & 8.470 & 25.23 \\
\hline SF & $0.996 \mathrm{ab}$ & 5.072 & 3.835 & 4.202 & 0.513 & 8.198 & 22.93 \\
\hline IN & $0.985 \mathrm{~b}$ & 5.111 & 3.852 & 4.223 & 0.449 & 8.459 & 23.25 \\
\hline \multicolumn{8}{|l|}{ Solución (S) } \\
\hline $\mathrm{S} 1$ & 1.005 & 5.122 & 3.874 & 4.225 & 0.506 & 8.346 & 23.51 \\
\hline S2 & 0.993 & 5.133 & 3.846 & 4.301 & 0.512 & 8.405 & 24.10 \\
\hline \multicolumn{8}{|l|}{ Factor } \\
\hline Injerto & $*$ & ns & ns & ns & ns & ns & ns \\
\hline Solución & ns & ns & ns & ns & ns & ns & ns \\
\hline I x S & ns & ns & ns & ns & ns & ns & ns \\
\hline
\end{tabular}

NI: plantas no injertadas; SF: plantas autoinjertadas; IN: plantas injertadas. Letras diferentes en una columna indican diferencias e.s. con

$\mathrm{p} \leq 0.05$. ns: no significativo; *: significativo $\mathrm{p} \leq 0.05 ; * *$ : significativo $\mathrm{p} \leq 0.01$

${ }^{(1)} \mathrm{La}$ acidez se expresa en $\mathrm{mL}$ de $\mathrm{OHNa} 0,1 \mathrm{~N} ;{ }^{(2)} \mathrm{El} \mathrm{Índice} \mathrm{de} \mathrm{madurez} \mathrm{es}{ }^{\circ} \mathrm{Brix} /$ Acidez; ${ }^{(3)} \mathrm{La}$ jugosidad se expresa en $\mathrm{mL} / 100 \mathrm{~g}$

\section{4.- DISCUSIÓN}

\section{1.- Injerto}

Los trabajos que han estudiado el efecto de distintos portainjertos sobre diferentes cultivares híbridos de tomate en los últimos años, han encontrado diferencias e.s. en la producción total, dando las plantas injertadas una mayor cosecha respecto de las plantas sin injertar. Estos resultados se basaron en que las plantas injertadas dieron un mayor número de frutos por planta y los frutos eran de media más pesados que los de las plantas sin injertar. (Khah et al., 2006; Gebologlu et al., 2011; Turham et al., 2011; Ibrahim et al., 2014).

En nuestro estudio no se vio un efecto del injerto sobre la producción total de la planta, ya que, aunque el peso medio total de los frutos procedentes de las plantas IN fue superior, coincidiendo con los resultados 
de los trabajos mencionados anteriormente, estas plantas dieron menos frutos en total que las plantas NI y que las plantas SF. Este hecho no coincide con los datos que ofrecen los trabajos anteriores, lo que tal vez pueda atribuirse a que nuestro experimento fue de corta duración.

Sin embargo, en la distribución de calibres sí se observó el efecto del injerto, dando estas plantas un mayor número de frutos de primera, con un peso superior a $200 \mathrm{~g}$, y menor número de tomates de segunda y tercera, entre 150 y $200 \mathrm{~g}$ y menos de $150 \mathrm{~g}$, respectivamente, lo cual supuso una diferencia en la producción en peso de estos calibres. Este resultado está en consonancia con los resultados de Rollón y Hoyos (2010) en su estudio de dos cultivares híbridos sobre tres portainjertos comerciales. En este trabajo, como en el presente, las plantas no injertadas dieron más frutos de calibres $\mathrm{M}$, que son equivalentes a nuestra clasificación de tercera, pues tienen un peso menor a $150 \mathrm{~g}$, respecto de las plantas injertadas, que además de dar menos frutos de tercera, dieron un número mayor de frutos de calibre GG, peso superior a $201 \mathrm{~g}$, equivalente a nuestros frutos de primera.

Este resultado se podría explicar por el número total de frutos de las plantas IN respecto a las NI. El aparato fotosintético de las plantas IN alimentó a un número menor de frutos, por lo que éstos alcanzaron un mayor peso, aumentando la producción de tomates de primera. Este resultado se puede comparar al estudio de Rahmatian et al. (2014) donde se condujeron plantas injertadas a uno o dos brazos, dando las plantas conducidas a un brazo un menor número de frutos pero con un peso superior al de las plantas conducidas a dos brazos, que produjeron un mayor número de frutos pero con un peso inferior.

Respecto al hecho de que en las plantas NI se obtuviera un mayor peso en frutos afectados por BER respecto de las plantas IN se podría explicar por la mejora en la eficiencia de toma tanto de agua como de nutrientes que el portainjerto utilizado ofrece a la variedad (Lykas et al., 2008), y en concreto en soslayar posibles efectos de estrés abiótico inducido por momentos puntuales de déficit hídrico o estrés salino por excesiva acumulación de sales en el sustrato.

El único parámetro de calidad que se vio afectado por el efecto del injerto en el presente trabajo fue la densidad del fruto, siendo menor en los frutos de las plantas IN respecto de las NI. Este hecho también se observó en el trabajo de Belda (2014) en melón donde los frutos de las plantas no injertadas fueron más densos que los de las plantas injertadas. 
Para el resto de parámetros no se encontraron diferencias entre tratamientos. Los resultados obtenidos en el trabajo de Barrett et al. (2012) sobre el efecto del injerto en una variedad tradicional de tomate coinciden con los nuestros. Ni los sólidos solubles ni la acidez titulable se vieron afectados por el uso o no de portainjerto en el manejo del cultivo. Estos dos factores y la proporción entre ellos en el fruto, índice de madurez, tienen un papel indispensable en el sabor y por tanto en la calidad del producto.

Respecto a la jugosidad y al porcentaje de materia seca no encontramos diferencias e.s., pero los frutos de las plantas NI dieron valores ligeramente superiores con respecto a los frutos de las plantas IN. Esto coincide con los resultados obtenidos en estos parámetros en el trabajo de Rollón (2010), salvo que en su caso las diferencias sí fueron e.s.

El efecto del injerto sobre estos y otros atributos de calidad han sido estudiados y los resultados obtenidos han sido variados, encontrando tanto una mejora en estos parámetros usando el injerto Ibrahim et al., (2014), como una disminución Turhan et al. (2011). También se encuentran trabajos en los que estos parámetros no se ven afectados, como Gebologlu (2011) o el nuestro propio. Esto demuestra que estos atributos dependen en gran medida del portainjerto utilizado, de la variedad y de buena combinación entre ambos.

\section{2.- Relación $\mathrm{N} / \mathrm{K}$}

No encontramos diferencias e.s. para la producción total pero, como ocurriera con el factor injerto, la distribución de los calibres si se vio influenciada por la solución nutritiva. La solución 2 (S2), con una proporción $\mathrm{N} / \mathrm{K}$ de 1.3 , dio más número de frutos de primera que la solución 1 (S1), N/K de 1.8, no habiendo diferencias para el resto de calibres.

Este resultado está en consonancia con lo obtenido en el trabajo de Hernández et al., (2009) en el que se comparaban los rendimientos de una variedad de tomate híbrido fertirrigados con cuatro soluciones nutritivas basadas en diferentes ratios $\mathrm{N} / \mathrm{K}$. La mejor relación fue 1:0.75, 1.3, ya que produjo más frutos de calibres extra y primera respecto de los otros tres tratamientos. Como en nuestro trabajo, la solución influyó en el tamaño, y por tanto en el peso de los frutos, coincidiendo en la proporción $\mathrm{N} / \mathrm{K}=1.3$.

En el caso de la fertilización en tomate no se puede generalizar que una proporción de nutrientes puede ser la más acertada, ya que la variedad es un factor definitivo a tener en cuenta a la hora de valorar los diferentes 
parámetros productivos. La existencia de multitud de variedades de tomate que se pueden cultivar hoy en día y el hecho de que cada una de ellas puede mostrar una extracción de nitrógeno y potasio diferente hace difícil la elección del ratio $\mathrm{N} / \mathrm{K}$ apropiado.

En el caso del cultivo de tomate valenciano, Pomares et al. (2009) en un estudio del IVIA en el que se compararon cuatro soluciones nutritivas, el ratio que dio mejores producciones fue $\mathrm{N} / \mathrm{K}=1.65$, que sería un ratio intermedio entre nuestras dos soluciones.

Además del ratio $\mathrm{N} / \mathrm{K}$, las dos soluciones se diferenciaron por la cantidad de cloruros presentes en ellas. La S2 contenía un nivel de cloruros más alto que la $\mathrm{S} 1$, ya que para lograr el ratio 1.3 se empleó el cloruro potásico como única fuente de potasio. Chapagain et al. (2003) en su estudio comparativo sobre la utilización de nitrato potásico frente al cloruro potásico no encontraron diferencias en la cosecha total de frutos, coincidiendo con los resultados del presente trabajo.

En el trabajo de Chapagain et al. (2003) la utilización de cloruros en la solución nutritiva afectó positivamente a la producción al reducir el número de frutos manchados o deformes. Si bien en nuestro trabajo no se redujo el número de frutos con fisiopatías, no hubo diferencias notables entre las dos soluciones para estos parámetros.

No encontramos influencia de la solución utilizada en ninguno de los parámetros de calidad estimados. Como en el trabajo de Hernádez et al. (2009) no se encontraron diferencias ni en materia seca, $\mathrm{pH}$, sólidos solubles ni en acidez titulable.

En el trabajo de Wright y Harris (1985) sí se encontraron diferencias en el contenido tanto de ácidos como de sólidos solubles utilizando un ratio fijo $\mathrm{N} / \mathrm{K}=0.6$, obteniendo niveles más altos conforme aumentaban las cantidades tanto de nitrógeno como de potasio. Con estos resultados parece observarse que a la hora de aumentar tanto los ácidos como los sólidos solubles podría ser más importante las cantidades absolutas, y por tanto la conductividad de la solución, que el ratio elegido.

Respecto a la utilización de cloruros, como ocurriera en el trabajo de Chapagain et al. (2003), ésta no afectó a la materia seca, ni al pH, ni a los sólidos solubles ni a la acidez titulable. 


\section{5.- CONCLUSIONES}

La combinación de "Maxifort", como portainjerto, y tomate "Valenciano", como variedad, en nuestras condiciones ha resultado provechosa en cuanto a que la producción de grandes calibres se ve aumentada. El mercado de este tipo de tomate aprecia los grandes calibres pagando por ellos un precio superior por kilogramo respecto de los calibres más bajos.

Respecto al equilibrio N/K en la formulación de la solución nutritiva para el cultivo de tomate "Valenciano" en nuestras condiciones, la proporción $\mathrm{N} / \mathrm{K}=1.3$ ha resultado válida a la hora de aumentar el porcentaje de frutos de primera, y como se dijo anteriormente, estos calibres se pagan mejor en el mercado. Del mismo modo parece interesante la utilización de cloruro potásico como fuente alternativa de fertilización potásica.

\section{REFERENCIAS BIBLIOGRÁFICAS}

AOAC Official Method 942.15. Acidity (Titratable) of Fruit Products. Official method of Analysis of AOAC International, ed. 18, 2005, Cap. 37, p.10.

Barrett, C., Zhao, X., Sims, C., Brecht, J., Dreyer, E., Gao, Z. 2012. Fruit Composition and Sensory Attributes of Organic Heirloom Tomatoes as Affected by Grafting. Hortechnology 22(6) 804- 809.

Belda, R. 2014.Comportamiento fisiológico y agronómico de las plantas injertadas de melón tipo piel de sapo. Trabajo Fin de Grado dirigido por Salvador Vicente López Galarza. Universitat Politècnica de València.

Cebolla, J. 2005. Recuperación de variedades tradicionales de tomate y pimiento. Caracterización y mejora genética. Tesis doctoral dirigida por Fernando Nuez. Universidad Politécnica de Valencia.

Chapagain, B.P., Wiesman, Z., Zaccai, M., Imas, P., Magen, H., 2003. Potassium chloride enhances fruit appearance and improves quality of fertirrigated greenhouse. Journal of plant Nutrition. Vol. 26 (3): 643-658.

Di Gioia, F.; Serio, F.; Buttaro, D.; Ayala, O.; Santamaria, P. 2010. Influence of rootstock on vegetable growth, fruit yield and quality in "cuore di bue", an heirloom tomato. J. Hort. Sci. Biotechnol. 85: 477-482 
Flores, F., Sánchez-Bel, P., Estañ, M., Martínez-Rodríguez, M., Moyano, E., Morales, B., Campos, J., Garcia-Abellán, J., Egea, M., Fernández-García, N., Romojaro, F., Bolarín, M. The effectiveness of grafting to improve tomato fruit quality. Scientia Horticulturae 125 (2010) 211-217.

Geboloğlu, N., Yılma E., Çakmak P., Aydın, M., Kasap, Y. 2011. Determining of the yield, quality and nutrient content of tomatoes grafted on different rootstocks in soilless culture. Sci.Res.Essays 6(10):2147-2153

Giner, A., Aguilar, J.M., Baixauli, C., Nuñez, A., Nájera, I., Maroto, J.V. 2011. Estudio comparativo de nuevas variedades de tomate grueso con resistencias a virosis, en ciclo de primavera. Fundación Ruralcaja Valencia Grupo CRM.

Hernández Díaz, M.I., Chailloux , M., Moreno, V., Ojeda, A., Julia Mirta Salgado, J.M.,Bruzón, O. 2009. Relaciones nitrógeno-potasio en fertirriego para el cultivo protegido del tomate en suelo Ferralítico Rojo. Pesq. agropec. bras., Brasília, v.44, n.5, p.429-436.

Hoyos, P. 2007. Situación del injerto en horticultura en España: especies, zonas de producción de plante, portainjertos. Horticultura, 199: $12-25$.

Ibrahim A., Wahb-Allah M., Abdel-Razzak H., Alsadon A., 2014. Growth, Yield, Quality and Water Use Efficiency of Grafted Tomato Plants Grown in Greenhouse under Different Irrigation Levels. Life Sci. J.;11(2):118- 126 .

Iqbal, M., Niamatullah, M., Yousaf, I., Munir, M., Zafarrullah, M. 2011. Effect of nitrogen and potassium on growth, economical yield and yield components of tomato. Sarhad J. Agric. 27(4): 545- 548.

Johnston, A y Milford, G. 2009. Nitrogen and potassium interactions in crops. The Potash Development Association. PO Box 697, York YO32 5WP, UK

Khah, E.M., Kakava, E.M., Mavromatis, A., Chachalis, D., Goulas, C. 2006. Effect of grafting on growth and yield of tomato (Lycopersicon esculentum Mill.) in greenhouse and open-field. Journal of Applied Horticulture, 8(1): 3-7.

Lee, J.M., Oda M. 2003. Grafting of herbaceous vegetable and ornamental crops. Hort.Rev. 28:61-124. 
Leonardi, C., Giuffrida, F., 2006. Variation of plant growth and macronutrient uptakein grafted tomatoes and eggplants on three different rootstocks. Eur. J. Hort. Sci.71, 97-101.

López, M., Gil, G. 2010. Ensayo de variedades de tomate "Valenciano". Cohoca Coop. V.

Lykas, C., Kittas, C., Zambeka, A. 2008. Water and fertilizers use efficiency in grafted and non grafted tomato plants on soilless culture. Acta Hort. 801:1551-1555.

Maroto J.V. 2002. Horticultura Herbácea Especial. Ediciones Mundiprensa. Madrid. 702 pp.

Miguel, A., 2011. El injerto en plantas de tomate. Serie Documentos. www.poscosecha.com/es/publicaciones/

Pomares, F., Aguilar, J.M., Baixauli, C., Giner, A., Nuñez, A., Juan, F., Nájera, I. 2009. Comportamiento agronómico de cuatro soluciones nutritivas, con tomate valenciano en ciclo de primavera. Centro de Fundación Ruralcaja, Paiporta (Valencia).

Rivard, C.L. and F.J. Louws. 2008. Grafting to manage soilborne diseases in heirloom tomato production. HortScience 43:2104- 2111.

Turhan A., Ozmen N., Serbeci M.S., Seniz V., 2011. Effects of grafting on different rootstocks on tomato fruit yield and quality. Hort. Sci. (Prague), 38: 142-149.

Zhang, F., Niu, F., Zhang, W. Chen, X., Li, C. Yuan, Y., Xie, J., 2010. Potassium nutrition of crops under varied regimes of nitrogen supply. Plant Soil. 335:21-34 